\title{
Differential Analysis of the LED Block Cipher*
}

\author{
Florian Mendel, Vincent Rijmen, Deniz Toz, and Kerem Varıcı \\ KU Leuven, ESAT/COSIC and IBBT, Belgium \\ \{florian.mendel, vincent.rijmen, deniz.toz, kerem.varici\}@esat.kuleuven.be
}

\begin{abstract}
In this paper, we present a security analysis of the lightweight block cipher LED proposed by Guo et al. at CHES 2011. Since the design of LED is very similar to the Even-Mansour scheme, we first review existing attacks on this scheme and extend them to related-key and relatedkey-cipher settings before we apply them to LED. We obtain results for 12 and 16 rounds (out of 32) for LED-64 and 16 and 24 rounds (out of 48) for LED-128. Furthermore, we present an observation on full LED in the related-key-cipher setting 1 . For all these attacks we need to find good differentials for one step (4 rounds) of LED. Therefore, we extend the study of plateau characteristics for AES-like structures from two rounds to four rounds when the key addition is replaced with a constant addition. We introduce an algorithm that can be used to find good differentials and right pairs for one step of LED. To be more precise, we can find more than $2^{10}$ right pairs for one step of LED with complexity of $2^{16}$ and memory requirement of $5 \times 2^{17}$. Moreover, a similar algorithm can also be used to find iterative characteristics for the LED.
\end{abstract}

\section{Introduction}

Security in embedded systems, such as RFID and sensor networks, where the area is restricted is getting more and more important since people started interacting with them in daily life more often. Improving the efficiency while preserving the security is one of the main challenges in this area and it has been an ongoing research problem. Recently, many algorithms have been developed to address this problem: hash functions like QUARK [1, PHOTON [13], SPONGENT [3] as well as block ciphers like Piccolo [23], LED [14], TWINE 24] and Klein [12. Each of them uses the advantage of the improved knowledge on the design and analysis of symmetric key components.

LED [14] is a lightweight block cipher proposed by Guo et al. at CHES 2011. While being dedicated to compact hardware implementation with one of the smallest area consumptions (among block ciphers with comparable parameters),

\footnotetext{
^ This work was sponsored he Research Fund KU Leuven, OT/08/027, by the IAP Programme P6/26 BCRYPT of the Belgian State (Belgian Science Policy) and by the European Commission through the ICT Programme under Contract ICT-2007216676 (ECRYPT II).

${ }^{1}$ Due to the page limitations, details of this part is given in the full version of the paper in 19 .
}

X. Wang and K. Sako (Eds.): ASIACRYPT 2012, LNCS 7658, pp. 190-207, 2012.

(C) International Association for Cryptologic Research 2012 
LED also offers reasonable performance in software. The design bears some resemblance with the (generalized) Even-Mansour construction [4] with the difference that the same key is used in each step for LED-64 or every second step in the case of the larger variant LED-128. The step function is based on AES-like design principles that provide good bounds against large classes of attacks including differential and linear cryptanalysis. Additionally, LED offers strong security arguments against attacks even in the related-key model.

To the best of our knowledge, no external analysis of LED with respect to differential cryptanalysis has been published so far. The best existing differential attacks are distinguishers for 15 (out of 32) rounds of LED-64 and 27 (out of 48 ) rounds of LED-128 in a hash setting, where the key is known to (or even chosen by) the attacker, described by the designers. Moreover, the security of LED against meet-in-the-middle attack has been investigated recently by Isobe et al. [16]. They describe attacks for 8 (out of 32) and 16 (out of 48) rounds for LED-64 and LED-128, respectively.

Our Contribution. In this paper, we present the first external cryptanalysis of LED with respect to differential cryptanalysis. First, we show attacks for LED-64 reduced to 12 and 16 rounds. Furthermore, we present an observation on full LED in the related-cipher setting 25. All our attacks are based on the attack of Daemen [5] on Even-Mansour construction [11 that is extended in a straightforward way to the related-key setting.

Secondly, we show how to improve the bound for the maximum expected differential probability (MEDP) for four rounds (one STEP) of LED from $2^{-32}$ to $2^{-41.75}$ using mega-boxes and the result of Park et al. [20].

Furthermore, we present algorithms to find differential characteristics with high probability that can be used in our attacks. By using the ideas of plateau characteristics [9] and extending the work with mega boxes [6], we are able to obtain characteristics for four rounds of LED. We find more than $2^{10}$ right pairs for a differential with a complexity less than $2^{16}$ time and $5 \times 2^{17}$ memory and an iterative characteristic with six right pairs with the same complexities. We emphasize that our method is not specific to the block cipher LED and it can be used in the analysis of any AES-like construction where the key addition is replaced with a constant addition.

Outline. This paper is organized as follows. In Section 2 we give a brief description of LED and introduce the required definitions for our analysis. In Section 3 . we describe the attacks on Even-Mansour construction and show how they can be extended to attack LED. We continue with differential analysis and give an algorithm to find the number of right pairs in a plateau characteristic in Section 4. We generalize this algorithm find characteristics for four rounds of LED in Section 5 and we provide the results for characteristics with high probability and iterative characteristics that can be used in our attacks in Section 6 . 


\section{Description of LED}

LED [14 is a conservative lightweight block cipher whose design can be seen as a special case of the generalized Even and Mansour construction [11] depicted in Figure 1.

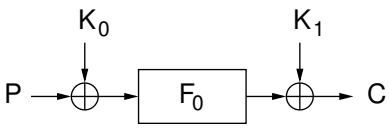

(a)

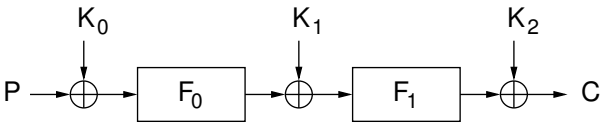

(b)

Fig. 1. Even-Mansour Construction with (a) $t=1$ and (b) $t=2$

LED accepts a 64 -bit plaintext $\mathrm{P}$, represented by a $4 \times 4$ array, and a 64 -bit (or 128-bit) user key as inputs, and is composed of 8 (or 12) STEP functions preceded by a key addition. The STEP function is an AES-like design composed of four rounds. Each round is combination of Constant addition, S-boxes, ShiftRows, and (a variant of) MixColumns. LED uses the PRESEnT S-box. In MixColumnsSerial, each column vector is multiplied by a matrix and replaced with the resulting vector. Note that the round constants for the second column are obtained from a linear shift register while the round constants for the remaining three columns do not change.

Key Schedule: LED has a simple key schedule where the 64-bit user key $K$ is used as it is in each round whereas the 128-bit key is divided into two parts, $K=K_{0} \| K_{1}$, and used alternately. For the remainder of this paper, we refer to these two versions as LED-64 and LED-128. For more detail, please check the specification of LED [14].

One observation is that the S-boxes and linear transformations in the round function of the cipher can be described by structure of a super box:

Definition 1 (Super box [9]). A super box maps an array a of $m$ elements $a_{i}$ to an array $e$ of $m$ elements $e_{i}$. Each of the elements has size $n$. A super box takes a key $k$ of size $m \times n=n_{b}$ where $n_{b}$ is the block size. It consists of the sequence of four transformations (layers): Substitution, Mixing, Round Key Addition, Substitution.

Similar to AES [7, two rounds of LED can also be described alternatively as four parallel instances of the LED super box where the key addition is replaced by the constant addition. So, instead of dealing with the classical 4-bit S-boxes, one can consider 16-bit super boxes each composed of two S-box layers surrounding one MixColumnsSerial (MC) and one AddConstants (AC) function.

Four rounds of LED can be described as a mega-box, where the elements are 16bit words and the LED super boxes defined above are seen as S-boxes. The linear transformation in the middle is a combination of ShiftRows, MixColumnsSerial and ShiftRows respectively. We will refer to this linear transformation as SMS. 


\section{Attacks on the Even-Mansour Construction and Application to LED}

The Even-Mansour construction is a simple and yet provably secure block cipher construction. The designers have shown that the number of queries needed to break the scheme is bounded by $2^{n / 2}$, where $n$ is the blocklength $(n=64$ for LED). A generic key recovery attack with chosen plaintexts showing that this bound is tight was introduced by Daemen [5]. Twenty years later, the construction was revisited. It was shown that the same bound applies to the known plaintext setting by using the slidex attack, an extended version of the slide attack [10].

Simultaneously, Bogdanov et al. generalized the construction in [4] to more steps and discussed its security. They even provided a security proof for the construction in the single-key setting. However, as pointed out by the authors, the scheme is insecure in the related-key setting. In this section, we focus on the attack of Daemen on the Even-Mansour construction, since it is the basis for all our attacks on LED. First we show how it can be extended to a related key attack on the generalized Even-Mansour construction. Then, we will use it to attack reduced versions of the LED block cipher.

\subsection{Daemen's Attack}

At Asiacrypt 1991 Daemen presented a generic key-recovery attack with complexity of $2^{n / 2}[5]$. It can be summarized as follows.

1. Choose a difference $\Delta$.

2. For $\ell$ values of $a$ compute $\Delta F_{0}=F_{0}(a) \oplus F_{0}(a \oplus \Delta)$ and save the pair $\left(\Delta F_{0}, a\right)$ in a list $L$.

3. Choose an arbitrary plaintext $P$ with $P^{\prime}=P \oplus \Delta$ and ask for the ciphertexts $C$ and $C^{\prime}$

4. Compute $\Delta C=C \oplus C^{\prime}$ and check if $\Delta C$ is in the list $L$ to get $a$.

- If $\Delta C$ is in the list $L$ then a candidate for the key is found. Compute $K_{0}=a \oplus P$ and $K_{1}=F_{0}(a) \oplus C$.

- Else go back to Step 3.

After repeating steps $3-4$ about $2^{n} / \ell$ times one expects to find the correct key with complexity of about $2^{n} / \ell+\ell$. Obviously the attack has the best complexity by choosing $\ell=2^{n / 2}$ resulting in a final attack complexity of about $2^{n / 2}$ and similar memory requirements.

Note that, the attack can be applied in an iterative way to attack the EvenMansour construction with $t>1$ with complexity of $2^{t \cdot n / 2}$ and similar memory requirements. For instance, if $t=2$ then we get a complexity of $2^{n}$.

\subsection{Using Daemen's Attack in a Related-Key Setting}

In certain scenarios one considers also related-key attacks where the adversary is allowed to get encryptions under several related keys. In this setting Daemen's 
attack can be adapted to attack $t$ steps of the Even-Mansour construction with complexity of $t \cdot 2^{n / 2}$ and similar memory requirements. For the sake of simplicity we first describe the attack for $t=2$.

Related Key Attack with $\boldsymbol{t}=\mathbf{2}$. Let $K, K^{\prime}$ be two related keys, where $K=K_{0}\left\|K_{1}\right\| K_{2}$ and $K^{\prime}=K_{0} \oplus \Delta_{0}\left\|K_{1} \oplus \Delta_{1}\right\| K_{2} \oplus \Delta_{2}$, with arbitrary (but known) $\Delta_{0}, \Delta_{1}, \Delta_{2}$ and $\Delta_{1} \neq 0$. Then we can do a key recovery attack on the Even-Mansour construction with $t=2$ with complexity of roughly $2^{n / 2}$ and similar memory requirements using the attack of Daemen [5]. It can be summarized as follows.

1. For $\ell$ values of $a$ compute $\Delta F_{1}=F_{1}(a) \oplus F_{1}\left(a \oplus \Delta_{1}\right)$ and save the pair $\left(\Delta F_{1}, a\right)$ in a list $L$.

2. Choose an arbitrary $P$ and $P^{\prime}=P \oplus \Delta_{0}$ and ask for the ciphertexts $C$ and $C^{\prime}$

3. Compute $\Delta C=C \oplus\left(C^{\prime} \oplus \Delta_{2}\right)$ and check if $\Delta C$ is in the list $L$ to get $a$.

- If $\Delta C$ is in the list $L$ then a candidate for $K_{2}$ is found, $K_{2}=F_{1}(a) \oplus C$.

- Else go back to Step 2.

After repeating steps $2-3$ about $2^{n} / \ell$ times, the expected number of matches in the list $L$ (i.e., candidates for $K_{2}$ ) is at least one. Note that, if we have more than one candidate for $K_{2}$ then we have to repeat the attack to get new candidates for $K_{2}$. The intersection of both sets of candidates gives us the correct key. Note that it is very unlikely that this intersection will have more than one solution.

Once $K_{2}$ is known one can apply the attack of Daemen to find $K_{0}$ and $K_{1}$. This results in a final attack complexity of about $2 \cdot 2^{n} / \ell+2 \ell$ and memory requirements of $\ell$. Again, the attack has the best complexity by choosing $\ell \approx 2^{n / 2}$ resulting in a final attack complexity of about $2 \cdot 2^{n / 2}$ and memory requirements of $2^{n / 2}$.

Related Key Attack with $\boldsymbol{t}>\mathbf{2}$. The related key attack can be extended to more steps by applying the attack for $t=2$ iteratively using more related keys with certain properties. Assume $t=3$ and there are two related keys $K=$ $K_{0}\left\|K_{1}\right\| K_{2} \| K_{3}$ and $K^{\prime}=K_{0} \oplus \Delta_{0}\left\|K_{1}\right\| K_{2} \oplus \Delta_{2} \| K_{3} \oplus \Delta_{3}$, with arbitrary (but known) $\Delta_{0}, \Delta_{2}, \Delta_{3}$ and $\Delta_{2} \neq 0$. Then one can find $K_{3}$ similar as in the attack on the Even-Mansour construction with $t=2$ with a complexity of roughly $2^{n / 2}$. Once $K_{3}$ is found one can apply the attack for $t=2$ with another pair of related keys to recover $K_{0}, K_{1}$ and $K_{2}$. In general, one can find the key for $t=i$ using $i$ related keys with certain properties.

\subsection{Attacks on Reduced LED}

In this section, we will discuss the application of the attacks described in the previous section to the LED block cipher. Due to the fact that in LED the same key is used more than once the number of steps that can be attacked is significantly reduced. However, the attack can still be used in a straightforward way to break one and two steps of LED-64 in a single-key and related-key setting, respectively. 
Both attacks have a complexity of about $2^{n / 2}$ and similar memory requirements. Note that a similar related-attack was described recently in [4].

However, both attacks can be extended to more steps in the case of LED-128. In more detail, we can attack four and six steps of LED-128 in the single-key and related-key setting, respectively. First, we describe an attack on four steps of LED128 based on Daemen's attack. It is based on the following simple observation (cf. Figure 2).

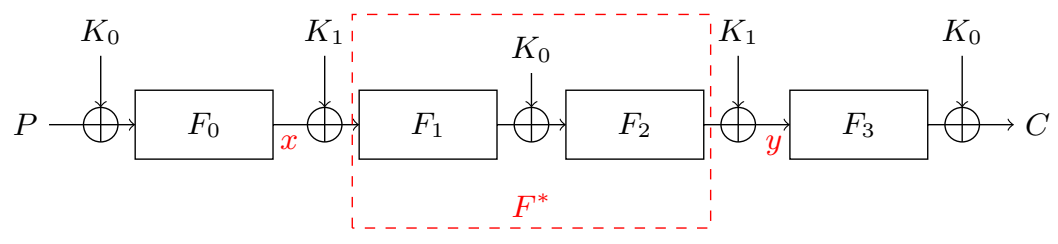

Fig. 2. Structure of LED-128 with $t=4$

Assume $K_{0}$ is known, then one can peel off the first and last key addition. Thus, the attacker can remove one iteration at each side of the cipher with a complexity of about $2^{64}$ tries on $K_{0}$. Moreover, assuming that $K_{0}$ is known two steps of LED-128 can be viewed as one big iteration using only $K_{1}$. In other words, we get a 'new' Even-Mansour construction with $t=1$ and one key $K_{1}$ where we can apply Daemen's attack to recover the key. Using this, one can find $K_{0}$ and $K_{1}$ for four steps of LED-128 with a complexity of about $2^{3 n / 2}$. It can be summarized as follows.

1. Guess the key $K_{0}$.

2. For $2^{n / 2}$ values $a$ and a fixed $\Delta$ compute $\Delta F^{*}=F^{*}(a) \oplus F^{*}(a \oplus \Delta)$ with $F^{*}(a)=F_{2}\left(F_{1}(a) \oplus K_{0}\right)$ and save the pair $\left(\Delta F^{*}, a\right)$ in a list $L$.

3. Choose an arbitrary $\mathrm{P}$ and compute $P^{\prime}=F_{0}^{-1}(x \oplus \Delta) \oplus K_{0}$ with $x=$ $F_{0}\left(P \oplus K_{0}\right)$. Ask for the ciphertexts $C$ and $C^{\prime}$.

4. Compute $\Delta y=y \oplus y^{\prime}$ with $y=F_{3}^{-1}\left(C \oplus K_{0}\right)$ and $y^{\prime}=F_{3}^{-1}\left(C^{\prime} \oplus K_{0}\right)$. Check if $\Delta y$ is in the list $L$ to get $a$.

- If $\Delta y$ is in the list $L$ then a candidate for the key is found. Compute $K_{1}=a \oplus x$.

- Else go back to Step 3.

5. Once $K_{1}$ is found check if the key $K=K_{0} \| K_{1}$ is correct.

Since the expected number of $K_{0}$ guesses that we need to make to find the correct key is $2^{n}$, we need to repeat the attack $2^{n}$ times. Since for each guess of $K_{0}$ we need about $2^{n / 2}$ computations to find $K_{1}$, the complexity of the attack is roughly $2^{3 n / 2}$. Note that the above attack needs the whole codebook. However, at the cost of a higher attack complexity, the data complexity of the attack can be reduced. To be more precise, in step 3 of the attack we can always choose $P$ from a predefined subset and when computing $P^{\prime}$ we check if it is also in this subset, if not then we repeat this step. Thus, the data complexity of the attack can be reduced by simultaneously increasing the time complexity. 
The attack can be extended to six steps of LED-128 using related keys as in the attack on the Even-Mansour construction with $t=2$. The attack is very similar as the attack on four steps. Basically only steps $2-4$ (Daemen's attack) are replaced by the related key attack described in the previous section. The result is a key-recovery attack on six steps (24 rounds) of LED-128 with complexity of about $2^{3 n / 2}$. Again, as in the attack on 4 steps the data complexity of the attack can be reduced on the cost of a higher attack complexity.

\subsection{Extending the Attack to More Steps}

In this section, we discuss how the attacks can be extended to more steps of LED. First, we show that by exploiting differential properties of the STEP-function $F$, it might be possible to extend the attacks on LED-64 by one or two steps. Moreover, the attack on 4 steps can also be used in related-cipher attack 25] with related key setting on full LED-128. We represent our observation in the full version of our paper [19].

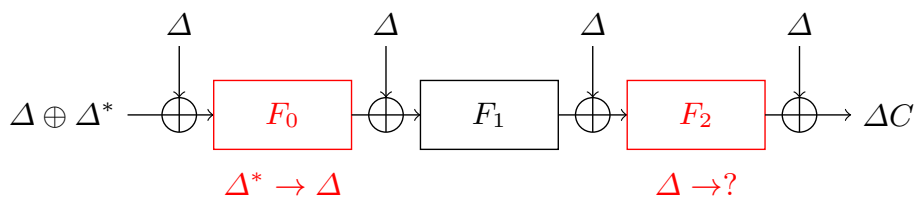

Fig. 3. Attack on LED-64 with $t=3$

In the following, we show how the attack can be extended to $t$ steps of LED64. The attack is based on the assumption that one can find a good related-key differential for the first $t-2$ steps such that one gets a zero difference after the key addition of step $t-2$. Then one can use Daemen's attack on the last 2 steps to recover the key. For In the attack on 3 steps we a differential with good probability in $F_{0}$ is used, see Figure 3. The attack can be summarized as follows.

1. Assume we have given two related keys $K_{0}$ and $K_{0}^{\prime}=K_{0} \oplus \Delta$ and furthermore the differential $\Delta^{*} \rightarrow \Delta$ for $F_{0}$ holds with probability $p \gg 2^{-64}$.

2. For $2^{\left(n+\frac{1}{p}\right) / 2}$ values $a$ compute $\Delta F_{2}=F_{2}(a) \oplus F_{2}(a \oplus \Delta)$ and save the pair $\left(\Delta F_{2}, a\right)$ in a list $L$.

3. Choose an arbitrary $P$ and $P^{\prime}=P \oplus \Delta^{*} \oplus \Delta$ and ask for the ciphertexts $C$ and $C^{\prime}$

4. Compute $\Delta C=C \oplus\left(C^{\prime} \oplus \Delta\right)$ and check if $\Delta C$ is in the list $L$ to get $a$.

- If $\Delta C$ is in the list $L$ then a candidate for $K_{0}$ is found, $K_{0}=F_{2}(a) \oplus C$.

- Else go back to step 3.

After repeating steps $3-4$ about $2^{\left(n+\frac{1}{p}\right) / 2}$ times, the expected number of matches in the list $L$ (and hence candidates for the key $K_{0}$ ) is $1 / p$. Since the differential in $F_{0}$ will hold with probability $p$, for one of these matches we will have $\Delta F_{1}=0$. 
Table 1. Summary of the attacks on LED

\begin{tabular}{l|rrrrr}
\hline algorithm & $\begin{array}{r}\text { \# STEP } \\
\text { functions }\end{array}$ & $\begin{array}{r}\text { time } \\
\text { complexity }\end{array}$ & $\begin{array}{r}\text { memory } \\
\text { complexity }\end{array}$ & attack type & reference \\
\hline LED-64 & 3 & $2^{\left(n+\frac{1}{p}\right) / 2}$ & $2^{\left(n+\frac{1}{p}\right) / 2}$ & related-key & Section 3.4 \\
& 4 & $2^{\left(n+\frac{1}{p}\right) / 2}$ & $2^{\left(n+\frac{1}{p}\right) / 2}$ & related-key & Section 3.4 \\
\hline LED-128 & 4 & $2^{3 n / 2}$ & $2^{n / 2}$ & single-key & Section [3.3 \\
& 6 & $2^{3 n / 2}$ & $2^{n / 2}$ & related-key & Section [3.3 \\
& 12 & $2^{\left(n+\frac{1}{p}\right) / 2}$ & $2^{\left(n+\frac{1}{p}\right) / 2}$ & related-key-cipher & {$[19$} \\
\hline
\end{tabular}

Hence, one will find the right key after testing all candidates for $K_{0}$ resulting from the $1 / p$ matches in the list $L$. The complexity and memory requirements of the attack depends on $p$, i.e. $2^{\left(n+\frac{1}{p}\right) / 2}$.

The attack on three steps can be extended to four steps of LED-64. Assume we can find a good iterative differential for $F_{1}$ that holds with probability $p$. Then this differential can be easily extended to a differential for the first 2 steps with the same probability (see Figure 4), resulting in an attack on 4 steps of LED-64 with complexity of $2^{\left(n+\frac{1}{p}\right) / 2}$ and similar memory requirements.

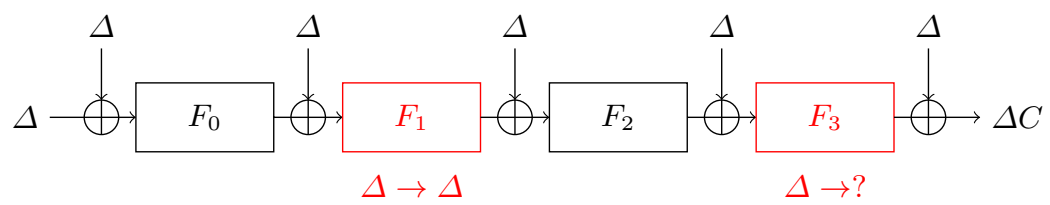

Fig. 4. Attack on LED-64 with $t=4$

In the Table1, we summarize the attacks on LED that are given in Section 3 , We will discuss in the following sections how to find good (iterative) differential characteristics for one step of LED that can be used in the attacks on three and four steps.

\section{Differential Analysis and Plateau Characteristics}

In this section, we start with some definitions that will be helpful to understand the rest of the paper. We then give an introduction of the previous work on AES [9] and describe how we can use this method to find two/four round characteristics efficiently (and the corresponding right pairs).

\subsection{Characteristics and Differentials}

Differential cryptanalysis 2] is one of the most powerful techniques used in analysis of block ciphers, hash functions, stream ciphers, etc. It investigates how 
an input difference (generally XOR) propagates through the target function. The concept of differential cryptanalysis starts with analyzing the components of the function, mostly focusing on S-boxes since they are the smallest nonlinear building block. In the analysis, we call an S-box active if it has a non-zero input difference, otherwise we call it passive.

A differential characteristic $Q=\left(\Delta_{0}, \Delta_{1}, \cdots, \Delta_{m}\right)$ is a sequence of differences through various stages of the encryption. The sequence consists of an input difference $\Delta_{0}$, followed by the output differences of all the steps $\left(\Delta_{1}, \Delta_{2}, \cdots, \Delta_{m}\right)$.

A differential [17] over a map is denoted by $\left(\Delta_{0}, \Delta_{m}\right)$ where $\Delta_{0}$ is the input difference and $\Delta_{m}$ is the output difference. The differential probability $\operatorname{DP}\left(\Delta_{0}, \Delta_{m}\right)$ of a differential over a map $f$ is the fraction of pairs with input difference $\Delta_{0}$ that have output difference $\Delta_{m}$.

For a keyed map, we can define differential probabilities $\operatorname{DP}[k]\left(\Delta_{0}, \Delta_{m}\right)$ and $\operatorname{DP}[k](Q)$ for each value $k$ of the key. Then, the expected differential probability (EDP) is the average of the differential probability over all keys. The weight of a differential or a characteristic is minus the binary logarithm of their EDP. Moreover, we define the height of a possible differential or a characteristic as the binary logarithm of the number of their right pairs satisfying $\left(\Delta_{0}, \Delta_{m}\right)$ for a fixed key.

A differential characteristic through the AES-like (including LED) super boxes consist of a sequence of four differences: the input difference $a$, the difference after the first substitution $b$, the difference after the mixing step which is equal to the difference after the round (key) constant addition $d$, and the output difference after the second substitution $e$. These characteristics are denoted by $Q=(a, b, d, e)$.

It can be shown that SMS is a map whose branch number is 5 . Therefore, a characteristic over a mega-box consists of 5 to 8 sub-characteristics, each over an LED super box. We denote the characteristics over the first and the second layer of super boxes by $(a, b, d, e)$ and $(f, g, i, j)$, respectively.

\subsection{The Maximum Expected Differential Probability of LED}

Differential cryptanalysis plays a crucial role in the analysis of symmetric key components since most of the cryptanalysis techniques are based on it. Therefore, giving bounds for resistance against differential cryptanalysis is one of the first steps in the evaluation of a design. In LED, the AES-like structure in the STEP function makes it possible to apply the previous work of [20] to bound the MEDP. By a straightforward computation of the formula stated in [20, Theorem 4], the designers compute the bound for the MEDP as $2^{-32}$. This bound can be improved by considering the STEP function as a mega-box and then using [20, Theorem 1] to bound the MEDP of LED as

$$
\max \left\{\max _{\substack{1 \leq i \leq 8 \\ 1 \leq x \leq 2^{16}-1}} \sum_{y=1}^{2^{16}-1}\left\{\mathrm{DP}^{\mathrm{sb}_{i}}(x, y)\right\}^{5}, \max _{\substack{1 \leq i \leq 8 \\ 1 \leq x \leq 2^{16}-1}} \sum_{y=1}^{2^{16}-1}\left\{\operatorname{DP}^{\mathrm{sb}_{i}}(y, x)\right\}^{5}\right\}=2^{-41.75} .
$$


Here $\mathrm{DP}^{\mathrm{Sb}_{i}}(x, y)$ is the probability of the characteristic $(x, y)$ for the $i$-th super box obtained from the Difference Distribution Table (DDT). This result improves the approximations used in [14, Table 1]. We provided the bound for the first STEP function; the results for the other super boxes are similar.

\subsection{Planar Differentials}

Let $\gamma$ be a map and let $F_{(a, b)}, G_{(a, b)}$ be the sets that contain the input values, respectively the output values, for the right pairs of the differential $(a, b)$. i.e., $F_{(a, b)}=\{x \mid \gamma(x)+\gamma(x+a)=b\}$ and $G_{(a, b)}=\gamma\left(F_{(a, b)}\right)$. A differential $(a, b)$ is called a planar differential, if $F_{(a, b)}$ and $G_{(a, b)}$ form affine subspaces $[9$. In that case, we can write:

$$
\begin{aligned}
F_{(a, b)} & =p+U_{(a, b)} \\
G_{(a, b)} & =q+V_{(a, b)},
\end{aligned}
$$

where $U_{(a, b)}$ and $V_{(a, b)}$ are uniquely defined vector spaces, $p$ any element in $F_{(a, b)}$ and $q$ any element in $G_{(a, b)}$. Note that, if a differential $(a, b)$ has exactly two or four right pairs, then it is always planar 9 .

Plateau characteristics [9] are a special type of characteristics whose probability for each value $k$ of the key, $D P[k](Q)$, depends on the key and can have only two values. For a fraction $2^{n_{b}-(\text { weight }(Q)+\text { height }(Q))}$ of the keys $D P[k](Q)=$ $2^{\text {height }(Q)-n_{b}}$ and for all other keys the it is zero. Note that the height is independent of the key.

Two-Round Plateau Characteristic Theorem states that a characteristic $Q=$ $(a, b, c)$ over a map consisting of two steps with a key addition in between, in which the differentials $(a, b)$ and $(b, c)$ are planar, is a plateau characteristic with $\operatorname{height}(Q)=\operatorname{dim}\left(V_{(a, b)} \cap U_{(b, c)}\right)$.

\subsection{Algorithm for Number of Right Pairs in a Plateau Characteristic}

Here, we describe the algorithm to find the number of right pairs of a given characteristic $Q=(a, b, d, e)$ through a super box. If the sub-characteristics $(a, d)$ and $(d, e)$ are planar then we can use the Two-Round Plateau Characteristic Theorem to compute the right pairs. Our aim in the algorithm is to build the matrix $B$ containing the basis vectors of $\left(M\left(V_{(a, b)}\right)\right)$ and $U_{(d, e)}$ where $M$ is the mixing operation and $M(V)=\{M(v) \mid v \in V\}$. We denote vectors by rows of $n_{b}$ bits.

The first step of our algorithm is to determine $V_{(a, b)}$ and $U_{(d, e)}$. Since, the super box is a set of $m$ parallel maps, $V_{(a, b)}$ and $U_{(d, e)}$ can be written as:

$$
\begin{aligned}
& V_{(a, b)}=V_{\left(a_{1}, b_{1}\right)} \times V_{\left(a_{2}, b_{2}\right)} \times \cdots \times V_{\left(a_{m}, b_{m}\right)} \\
& U_{(d, e)}=U_{\left(d_{1}, e_{1}\right)} \times U_{\left(d_{2}, e_{2}\right)} \times \cdots \times U_{\left(d_{m}, e_{m}\right)}
\end{aligned}
$$

by using the Lemma 4 in $\left[9\right.$. Now, if $\left|G_{\left(a_{i}, b_{i}\right)}\right|>0$, we are interested in the output values of the right pairs. 
- If $\left|G_{\left(a_{i}, b_{i}\right)}\right|=2$, then the right pairs have input values in the set $\left\{q+\left\{0, b_{i}\right\}\right\}$ for some $q$ in $G_{\left(a_{i}, b_{i}\right)}$, the basis vector for $V_{\left(a_{i}, b_{i}\right)}$ being $b_{i}$.

- If $\left|G_{\left(a_{i}, b_{i}\right)}\right|=2^{k}$ where $2 \leq k<n$, then $V_{\left(a_{i}, b_{i}\right)}=<b_{i}, \beta_{i}^{1}, \ldots, \beta_{i}^{k}>$ and hence $V_{\left(a_{i}, b_{i}\right)}$ is said to be spanned by $b_{i}$ and $\beta_{i}^{j}$ 's.

- If $\left(a_{i}, b_{i}\right)=(0,0)$ then $G_{\left(a_{i}, b_{i}\right)}$ covers the whole space and $V_{\left(a_{i}, b_{i}\right)}=<$ $w_{0}, w_{1}, \cdots, w_{n-1}>$ where $w_{j}$ is a coordinate vector (i.e. a vector with 1 at position $j$ and zero at all other positions) and $V$ is the standard basis.

Similarly, if $\left|F_{\left(d_{i}, e_{i}\right)}\right|>0$, we are interested in the input values of the right pairs. When we find the right pairs for each parallel map we can compute the height by using Algorithm [1. The number of dependent rows in $B$ gives $\operatorname{dim}\left(M\left(V_{(a, b)}\right) \cap\right.$ $\left.U_{(d, e)}\right)$ which is equal to the height.

Algorithm 1 calls the following subroutines. $\operatorname{Add}(v)$ adds the vector $v$ as a new row to the matrix B. RowReduce is the Gaussian Elimination and RowCount gives the number of nonzero rows of a matrix.

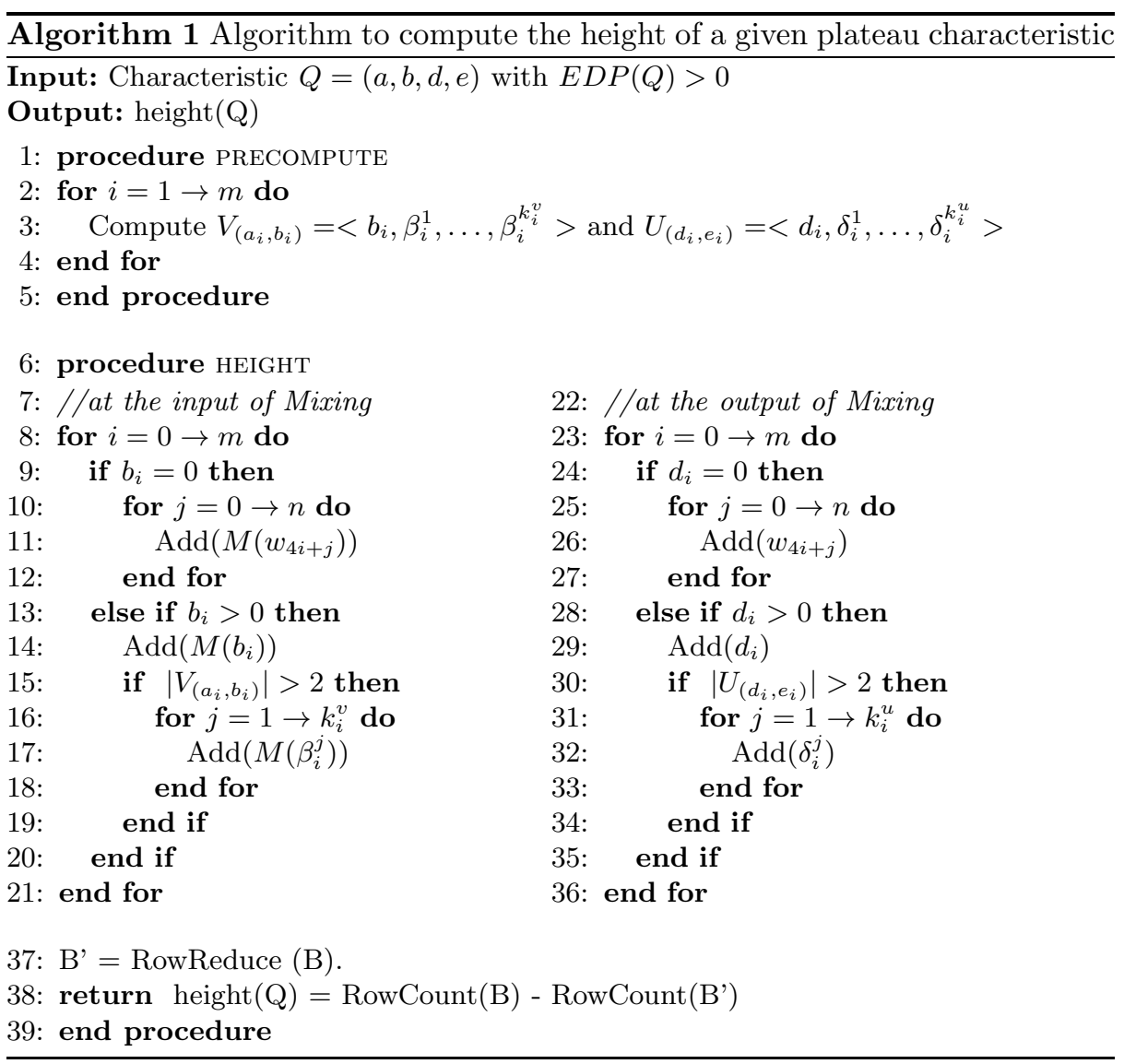


The algorithm also gives us an insight on how to find the right pairs which can be determined by intersecting the affine spaces $F_{(a, b)} \cap\left(G_{(b, c)} \oplus k\right)$. This can be efficiently done by preparing the set of linear equations to solve. We would like to emphasize that, for a fraction of the keys the right pairs exists and their values differ depending on the key. On the other hand, if the constant operation is used instead of the key addition operation in the cipher, then it is not guaranteed always to have a solution.

\section{Non-plateau Characteristics: LED Mega-Box}

As we mentioned in Section 2 two rounds of LED can be considered as a super box and four rounds is defined as a mega-box. Let $(a, b, d, e)$ and $(f, g, i, j)$ denote the characteristic through the super boxes at the input and the output of SMS respectively. Since the super boxes are key independent, we consider them as 16 -bit S-boxes. This allows us to omit the middle values $(b, d)$ and $(g, i)$ and use the differentials $(a, e)$ and $(f, j)$ in our analysis.

In order to use the two-round plateau characteristic theorem, it is required that the set of output values $G_{(a, e)}$ and the set of input values $F_{(f, j)}$ for the right pairs must be affine spaces/planar. However, this is not always guaranteed when the number of right pairs is greater than 4 . Although the difference between the values of each pair is known and constant, some extra conditions between the pairs are also required for a set to become affine/planar. Therefore, we have to work with a union of affine spaces in order to compute the number of right pairs of a given characteristic. In the following, we will denote by height* the binary logarithm of the maximum number of right pairs of a given characteristic, over all values of the key. For a plateau characteristic, height* equals the height.

The details of our algorithm are given below. An algorithmic description can be found in Algorithm 2 ,

Precomputation: The first step of our algorithm is finding $G_{(a, e)}$ and $F_{(f, j)}$ for the given path, and the next step is obtaining the subspace decompositions of $V_{(a, e)}$ and $U_{(f, j)}$. If $V_{\left(a_{i}, e_{i}\right)}$ is affine then $V_{\left(a_{i}, e_{i}\right)}=<e, \varepsilon_{1}, \ldots, \varepsilon_{n}>$, otherwise it is a union of smaller vector spaces, i.e. $V_{\left(a_{i}, e_{i}\right)}=V_{\left(a_{i}, e_{i}\right)}^{1} \cup V_{\left(a_{i}, e_{i}\right)}^{2} \cup \ldots V_{\left(a_{i}, e_{i}\right)}^{m}$ where $m \geq 2$. Therefore, we have to find the corresponding basis vectors $\left(\varepsilon_{i}\right.$ 's $)$ for each subspace. The results are then stored in a list, $L_{i}$, for each active super box.

Analysis: We then use the Two-Round Plateau Characteristic Theorem to compute the height using the basis vectors obtained in the precomputation phase. Since the solution exist only for a fraction of the constant values, we check whether the given round constant is in the solution set or not. This step can also be done by solving a system of linear equations as in two-rounds, but this time the equations are obtained from the SMS layer and the basis vectors of the super boxes.

Here, we would like to emphasize that the solution does not always exist for the round constant of LED. Denote by $K_{q}$, the set of values, $k$, such that 


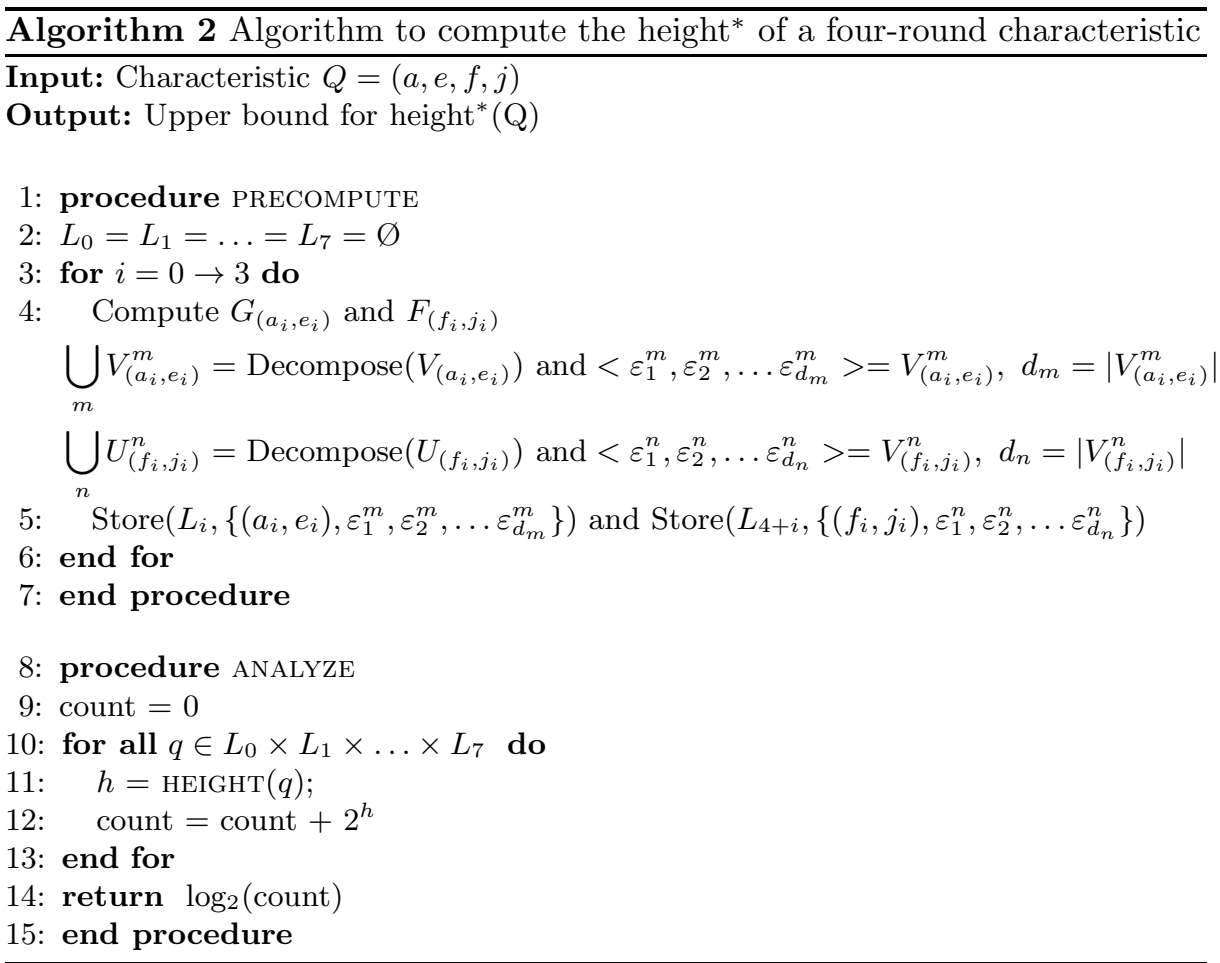

$\operatorname{DP}[k](q)>0$. Since constants are used in the round function of LED, it is not guaranteed that the round constant, $c_{r} \in K_{q}$ for all $q$. Therefore, the algorithm gives an upper bound for height* $(Q)$. If the key addition was used in the round function rather than constant addition, it could be possible to find a key value $k \in K_{q}$ for all $q$ satisfying the upper bound.

On the other hand, if the key addition was used, the Algorithm 2 could not be applied immediately, since the lists $L_{i}$ would depend on the key values and would not be unique. This would require recomputation of the lists for each key value increasing the complexity of the algorithm.

Note that, since height* for four rounds is the summation over all possible decompositions $q \in L_{0} \times L_{1} \times \ldots \times L_{7}$ of the characteristic $Q$, height* $(Q)$ is not guaranteed to be an integer, although height $(q)$ is integer for all $q$.

In Algorithm 2, Store adds input/output differences and the basis vectors $\left\{\varepsilon_{1}, \varepsilon_{2}, \ldots\right\}$ to the list $L$. HEIGHT is given in Algorithm 1 used with parameters $m=4$ and $n=16$.

\section{Application of the Algorithms 1 and 2}

In this section, we give two examples to demonstrate how Algorithm 1 and Algorithm 2 work. These examples can directly be used with attacks described 


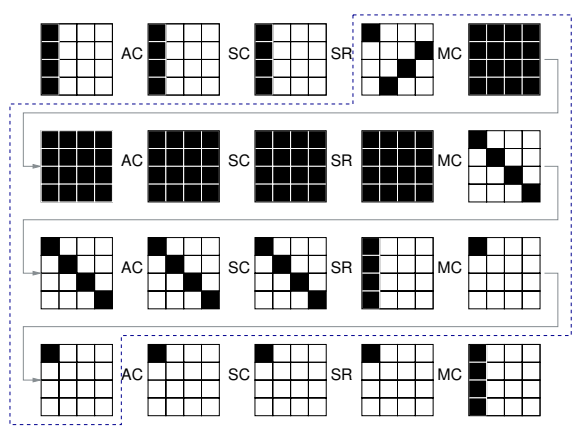

(a)

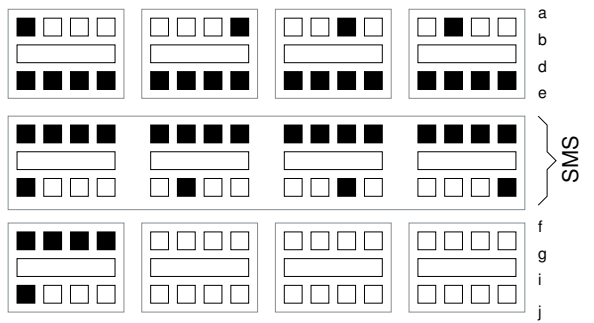

(b)

Fig. 5. (a)Path for iterative characteristics of the LED cipher (b)Mega-box representation of the same path

in Section 3.4. We do not claim that these are the best characteristics in terms of probability for the STEP function of LED that one can find. For both examples, we fix the number of active S-boxes to 25 for four rounds of LED. Since, we know from previous work 9] that all the characteristics with high probability are expected to have a low weight and a low number of active S-boxes. This also allows us to reduce the time and memory complexities of our algorithm and make the computation feasible.

\subsection{Iterative Characteristics}

Our aim is to find iterative characteristics (i.e., characteristics that have the same input and output difference) for the STEP function of the LED block cipher. We show that it is possible to obtain multiple iterative characteristics by using the 16-bit boxes and the two round plateau characteristic theorem in $2^{16}$ time and around $5 \times 2^{17}$ memory. In terms of efficiency, this computation can be compared with the inbound technique of the rebound attack [18. The main advantage of our computation is that many characteristics can be found whereas with the rebound attack, the expected number of characteristics that we find, equals one, using the same time complexity and slightly less data complexity.

In our analysis we used the differential path given in Figure 5. It is possible to adopt the algorithm for the other possible differential paths. The algorithm is summarized as follows:

Precomputation: For each of the active super boxes, obtain the differentials $\left(a_{i}, e_{i}\right)$ (or $\left.\left(f_{i}, j_{i}\right)\right)$ for the given path and find the corresponding right pairs $G_{\left(a_{i}, e_{i}\right)}$ (or $\left.F_{\left(f_{i}, j_{i}\right)}\right)$. Then compute their affine subspace decomposition and the corresponding basis vectors. Store the input/output differences together with the basis vectors in a list. We denote these lists as $L_{0}, L_{1}, L_{2}, L_{3}$ for the super boxes at the input and $L_{4}$ for the super box at the output of the SMS layer. Note that this calculation is done for all possible differentials. Each list has around $2^{17}$ elements, therefore the total memory requirement of this step is $5 \times 2^{17}$. 


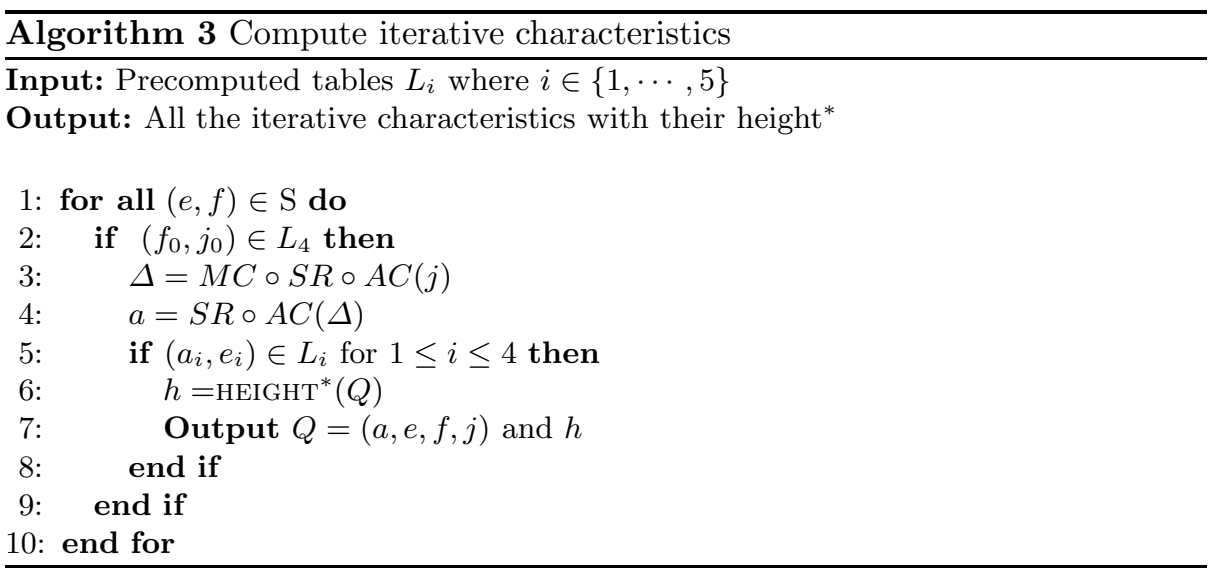

Analysis: We start from the four MixColumnsSerial operations in the SMS layer. Each of them has only one 4-bit word active at the output, hence we have $15^{4} \approx 2^{16}$ possibilities for the differences at $f$ (call the set of possibilities $S$ ). For each of these differences, we obtain the possible differences at $j$ by using the precomputed list $L_{4}$. Then, we compute $(M C \circ S R \circ A C)(j)=\Delta$ which is the output difference after four rounds of the STEP function and is also equal to the input difference of the STEP function since we are interested in iterative characteristics. We make one more computation $(S R \circ A C)(\Delta)$ to obtain the difference at $a$. Note that by choosing a difference for $f$, we have already fixed the difference at $e$. We then check whether $\left(a_{i}, e_{i}\right)$ is in the list $L_{i}$ for $0 \leq i \leq 3$. If it does for all $i$, we use the Algorithm 2 to compute the height* and find the right pairs.

Results: In our analysis we found 240 iterative characteristics for the pattern given in Figure 5 but not all of them have a solution for the round constants of the LED block cipher. One of these characteristics is given below. It has 6 right pairs and the corresponding right pairs are given in [19].

\begin{tabular}{c|cccc}
\hline$a$ & $0 \times 6000$ & $0 \times 0003$ & $0 \times 0070$ & $0 \times 0 C 00$ \\
$e$ & $0 \times 6962$ & $0 \times 5848$ & $0 \times 46 A 3$ & $0 \times 5 C B F$ \\
\hline$f$ & $0 \times 943 C$ & $0 \times 0000$ & $0 \times 0000$ & $0 \times 0000$ \\
$j$ & $0 \times 8000$ & $0 \times 0000$ & $0 \times 0000$ & $0 \times 0000$ \\
\hline
\end{tabular}

\subsection{Characteristics with High Height*}

In this section, our aim is to find characteristics with high height* for the STEP function of the LED block cipher. We show that it is possible to obtain such characteristics by using a similar algorithm to Algorithm 3 with $2^{16}$ time complexity and $5 \times 2^{17}$ memory complexity. In our analysis we focused on the differential path given in Figure 6 and searched for characteristics whose height* is greater than 5 . 


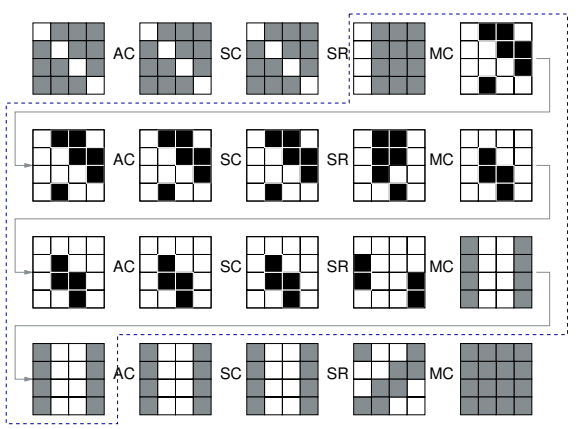

(a)

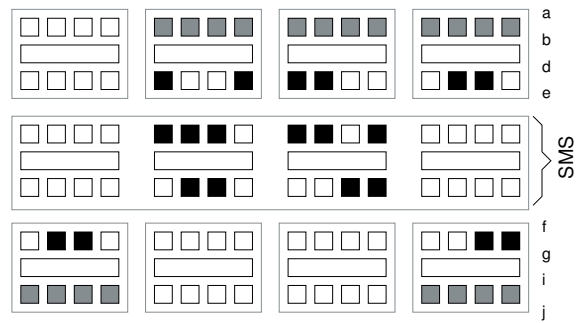

(b)

active word $\square$ passive word $\square$ active / passive word

Fig. 6. (a)Characteristics of the LED cipher with high height* (b)Mega-box representation of the same characteristics

Precomputation: All possible differentials together with the basis vectors of their affine space decomposition are stored in the lists $L_{1}, L_{2}, L_{3}$ for each of the the super boxes at the input and in the lists $L_{4}, L_{7}$ for the super boxes at the output of the SMS layer. Again, each list has around $2^{17}$ elements, and the total the memory requirement of this step is $5 \times 2^{17}$.

Analysis: We start from the two active MixColumnsSerial operations in the SMS layer. Each of them has two 4-bit words active at the output, hence we have $\left(15^{2}\right)^{2} \approx 2^{16}$ possibilities for the differences at $f$. For each of these possibilities, we obtain the possible differences at $a$ by using the precomputed lists $L_{1}, L_{2}$ and $L_{3}$. Similarly, the possible differences at $j$ are obtained by using the lists $L_{4}$ and $L_{7}$. We then use Algorithm 2 to compute the height and find the right pairs.

Results: Assume that $\operatorname{dim}\left(V_{(a, e)}\right)>0$ and $\operatorname{dim}\left(U_{(f, j)}\right)>0$, then we can write $V_{(a, e)}=\bigcup_{m} V_{(a, e)}^{m}$ and $U_{(f, j)}=\bigcup_{n} U_{(f, j)}^{n}$. We define a partition by $Q_{m n}$ where $Q_{m n}=S M S\left(V_{(a, e)}^{m}\right) \cap U_{(f, j)}^{n}$. Then we know that height* $(Q) \leq \log _{2}\left(\sum_{m, n} 2^{\operatorname{dim}\left(Q_{m n}\right)}\right)$ (see Algorithm 2). In our analysis we observed that it is not easy to find a partition whose height is greater than six, but by combining all partitions, we were able to find characteristics which have height* greater than eleven or twelve. One example of such characteristics is provided below.

\begin{tabular}{c|cccc}
\hline$a$ & 0x0000 & 0x0F91 & 0x2F0B & 0x2803 \\
$e$ & 0x0000 & 0xC00D & 0x8F00 & 0x0F50 \\
\hline$f$ & 0x0CD0 & 0x0000 & 0x0000 & 0x00C8 \\
$j$ & 0x8C07 & 0x0000 & 0x0000 & 0x50BF \\
\hline
\end{tabular}

The upper bound for height* is computed as 12.16 by using the formula. However, not all partitions have a solution for the given round constant, and we obtain only 1026 right pairs for the round constants used in LED. We also 
computed the number of right pairs by changing the round constant used in round three of the STEP function. The number of right pairs is computed as $1024 \pm \epsilon$ where $\epsilon \leq 116$ for all constants.

To sum up, we introduced not only a new method that can be useful in the security evaluation of AES-like structures but we also showed that by using this method it is possible to obtain characteristics that can be used to attack LED (see Section 3.4).

\section{Future Work and Open Problems}

The analysis of super boxes and mega-boxes play an important role in the cryptanalysis of AES-like ciphers. In this paper, we focused on characteristics for the block cipher LED with 25 active S-boxes. Since it is not feasible to compute the whole distribution of the characteristics for four rounds of LED, we focus only on characteristics that may have many right pairs. Therefore, our results cover characteristics with high height* and iterative characteristics with a fixed pattern. The examples given in this paper are the best ones that we computed. But still, it is possible to cover other patterns with 25 active S-boxes and they might give better results and at the same time result in improvements of our attacks.

We want to note that the algorithms given in this paper can also be used to compute the differentials for constructions using four rounds of AES as internal building block such as Pelican [8] giving new insights on these designs. Moreover, these algorithms might also be used in the computation of the inbound phase of the rebound attack.

\section{References}

1. Aumasson, J.-P., Henzen, L., Meier, W., Naya-Plasencia, M.: QuARK: A Lightweight Hash. In: Mangard, S., Standaert, F.-X. (eds.) CHES 2010. LNCS, vol. 6225 , pp. 1-15. Springer, Heidelberg (2010)

2. Biham, E., Shamir, A.: Differential Cryptanalysis of DES-like Cryptosystems. In: Menezes, A., Vanstone, S.A. (eds.) CRYPTO 1990. LNCS, vol. 537, pp. 2-21. Springer, Heidelberg (1991)

3. Bogdanov, A., Knezevic, M., Leander, G., Toz, D., Varıcı, K., Verbauwhede, I.: SPONGENT: A Lightweight Hash Function. In: Preneel, Takagi [22], pp. 312-325

4. Bogdanov, A., Knudsen, L.R., Leander, G., Standaert, F.X., Steinberger, J.P., Tischhauser, E.: Key-Alternating Ciphers in a Provable Setting: Encryption Using a Small Number of Public Permutations - (Extended Abstract). In: Pointcheval, Johansson [21], pp. 45-62

5. Daemen, J.: Limitations of the Even-Mansour Construction. In: Imai, et al. [15], pp. $495-498$

6. Daemen, J., Lamberger, M., Pramstaller, N., Rijmen, V., Vercauteren, F.: Computational aspects of the expected differential probability of 4-round AES and AES-like ciphers. Computing 85(1-2), 85-104 (2009)

7. Daemen, J., Rijmen, V.: The Design of Rijndael: AES - The Advanced Encryption Standard. Springer (2002) 
8. Daemen, J., Rijmen, V.: The Pelican MAC Function. IACR Cryptology ePrint Archive 2005, 88 (2005)

9. Daemen, J., Rijmen, V.: Plateau characteristics. IET Information Security 1(1), 11-17 (2007)

10. Dunkelman, O., Keller, N., Shamir, A.: Minimalism in Cryptography: The EvenMansour Scheme Revisited. In: Pointcheval, Johansson [21], pp. 336-354

11. Even, S., Mansour, Y.: A Construction of a Cipher From a Single Pseudorandom Permutation. In: Imai, et al. [15], pp. 210-224

12. Gong, Z., Nikova, S., Law, Y.W.: KLEIN: A New Family of Lightweight Block Ciphers. In: Juels, A., Paar, C. (eds.) RFIDSec 2011. LNCS, vol. 7055, pp. 1-18. Springer, Heidelberg (2012)

13. Guo, J., Peyrin, T., Poschmann, A.: The PHOTON Family of Lightweight Hash Functions. In: Rogaway, P. (ed.) CRYPTO 2011. LNCS, vol. 6841, pp. 222-239. Springer, Heidelberg (2011)

14. Guo, J., Peyrin, T., Poschmann, A., Robshaw, M.J.B.: The LED Block Cipher. In: Preneel, Takagi [22], pp. 326-341

15. Imai, H., Rivest, R.L., Matsumoto, T.: ASIACRYPT 1991. LNCS, vol. 739. Springer, Heidelberg (1993)

16. Isobe, T., Shibutani, K.: Security Analysis of the Lightweight Block Ciphers XTEA, LED and Piccolo. In: Susilo, W., Mu, Y., Seberry, J. (eds.) ACISP 2012. LNCS, vol. 7372, pp. 71-86. Springer, Heidelberg (2012)

17. Lai, X., Massey, J.L., Murphy, S.: Markov Ciphers and Differential Cryptanalysis. In: Davies, D.W. (ed.) EUROCRYPT 1991. LNCS, vol. 547, pp. 17-38. Springer, Heidelberg (1991)

18. Mendel, F., Rechberger, C., Schläffer, M., Thomsen, S.S.: The Rebound Attack: Cryptanalysis of Reduced Whirlpool and Grøstl. In: Dunkelman, O. (ed.) FSE 2009. LNCS, vol. 5665, pp. 260-276. Springer, Heidelberg (2009)

19. Mendel, F., Rijmen, V., Toz, D., Varıcı, K.: Differential Analysis of the LED Block Cipher. Cryptology ePrint Archive, Report 2012/544 (2012), http://eprint.iacr.org/

20. Park, S., Sung, S.H., Lee, S., Lim, J.: Improving the Upper Bound on the Maximum Differential and the Maximum Linear Hull Probability for SPN Structures and AES. In: Johansson, T. (ed.) FSE 2003. LNCS, vol. 2887, pp. 247-260. Springer, Heidelberg (2003)

21. Pointcheval, D., Johansson, T. (eds.): EUROCRYPT 2012. LNCS, vol. 7237, pp. 2012-2031. Springer, Heidelberg (2012)

22. Preneel, B., Takagi, T. (eds.): CHES 2011. LNCS, vol. 6917, pp. 2011-2013. Springer, Heidelberg (2011)

23. Shibutani, K., Isobe, T., Hiwatari, H., Mitsuda, A., Akishita, T., Shirai, T.: Piccolo: An Ultra-Lightweight Blockcipher. In: Preneel, Takagi [22], pp. 342-357

24. Suzaki, T., Minematsu, K., Morioka, S., Kobayashi, E.: Twine: A Lightweight, Versatile Blockcipher. In: ECRYPT Workshop on Lightweight Cryptography (2011), http://www.uclouvain.be/crypto/ecrypt_lc11/static/post_proceedings.pdf

25. Wu, H.: Related-Cipher Attacks. In: Deng, R.H., Qing, S., Bao, F., Zhou, J. (eds.) ICICS 2002. LNCS, vol. 2513, pp. 447-455. Springer, Heidelberg (2002) 